\title{
Resección hepática en metástasis de cáncer colorrectal, análisis de supervivencia de una cohorte de pacientes en el Hospital Pablo Tobón Uribe
}

\author{
Juan Javier Agudelo ${ }^{1,2}$, Jaime Chávez ${ }^{3}$, Álvaro Duarte ${ }^{3}$, Sergio Hoyos ${ }^{2,3,4,5}$
}

Palabras clave: neoplasias; hígado; neoplasias del colon; metástasis de la neoplasia; hepatectomía; supervivencia.

\section{Resumen}

Introducción. La resección hepática sigue siendo la única opción de tratamiento con intención curativa y con posibilidad de supervivencia a largo plazo. El objetivo del estudio fue evaluar los resultados de la resección hepática en un grupo de pacientes.

Métodos. Se llevó a cabo un estudio descriptivo retrospectivo de una serie consecutiva de 97 hepatectomías por metástasis de cáncer colorrectal, en el periodo comprendido entre el 15 de junio de 2005 y el 30 diciembre de 2015, en el Hospital Pablo Tobón Uribe de Medellín. Se hizo análisis bivariado de los factores asociados a la supervivencia.

Médico, residente de Cirugía General, Universidad CES, Medellín, Colombia

2 Médico, Grupo de Gastrohepatología, Facultad de Medicina, Universidad de Antioquia, Medellín, Colombia

3 Médico, Cirugía Hepatobiliar y Pancreática, Hospital Pablo Tobón Uribe, Medellín, Colombia

4 Médico, Grupo de Epidemiología, Universidad CES, Medellín, Colombia

5 Médico, Programa de Trasplante Hepático, Hospital Pablo Tobón Uribe, Universidad de Antioquia, Medellín, Colombia

Fecha de recibido: 6 de marzo de 2017

Fecha de aprobación: 17 de abril de 2017

Citar como: Agudelo JJ, Chávez J, Duarte A, Hoyos S. Resección hepática en metástasis de cáncer colorrectal, análisis de supervivencia de una cohorte de pacientes en el Hospital Pablo Tobón Uribe. Rev Colomb Cir. 2017;32:186-92.
Resultados. De 370 hepatectomías, se practicaron 97 resecciones hepáticas en 93 pacientes por metástasis de cáncer colorrectal. El promedio de edad fue de 58 años, $y$ el 50,5\% fueron resecciones hepáticas mayores. La mediana del volumen de sangrado intraoperatorio fue de $200 \mathrm{ml}$ (rango intercuartílico, RIQ: 100 a $400 \mathrm{ml}$ ) y la mediana del tiempo quirúrgico fue de 150 minutos (RIQ: 120 a 180 minutos). La estancia hospitalaria tuvo una mediana de 3 dias (RIQ: 2 a 4 dias). Solo se trasladó a la unidad de cuidados intensivos el 25,9\% de los pacientes; se presentaron complicaciones en $21,5 \%$ de los pacientes. La mortalidad perioperatoria a 90 días fue de 2,06\%. Se cumplió con el protocolo quirúrgico de recuperación rápida (Fast Track Surgery) en 54,8\% de los pacientes. La supervivencia global a cinco años fue de 34,5\% y, a 100 meses, de $23 \%$. Debido a que no se encontró significancia estadística con ninguna de las variables en el análisis bivariado para supervivencia, no se hizo un análisis multivariado.

Conclusiones. La resección hepática en pacientes con metástasis de cáncer colorrectal se puede practicar en nuestro medio de manera segura, con morbilidad, mortalidad y resultado oncológico comparables a los de otras series del mundo.

\section{Introducción}

El cáncer colorrectal se ha convertido en la tercera neoplasia maligna más común y es la cuarta causa de mortalidad por cáncer en el mundo. De 20 a $25 \%$ de los pacientes con cáncer colorrectal se presentan en estadio 
IV en el momento del diagnóstico, el $25 \%$ presentará metástasis hepáticas sincrónicas y el $50 \%$ desarrollará metástasis hepáticas durante el curso de su enfermedad. Las metástasis hepáticas causan la muerte en dos tercios de los pacientes que las presentan, en $80 \%$ de los casos no son resecables en el momento de su diagnóstico y de 50 a $60 \%$ de las recurrencias tumorales se presentan en el hígado. El pronóstico para los casos no tratados es pobre, con una supervivencia menor de $5 \%$ a los cinco años después de su diagnóstico ${ }^{1}$.

La resección hepática sigue siendo la única opción de tratamiento con intención curativa y con posibilidad de supervivencia a largo plazo para este grupo de pacientes; en las series modernas se reporta una supervivencia global a cinco años de 25 a $44 \%{ }^{2,3}$.

No existen en nuestro medio series publicadas que permitan conocer los resultados perioperatorios y oncológicos a largo plazo, de los pacientes sometidos a resección hepática por metástasis de cáncer colorrectal. En el presente estudio se describen los resultados obtenidos con la resección hepática de metástasis de cáncer colorrectal, en la Unidad de Cirugía Hepatobiliar y Pancreática del Hospital Pablo Tobón Uribe de Medellín. Se describen los factores asociados a la mortalidad perioperatoria (90 días) de estos pacientes. Los objetivos específicos fueron describir los factores clínicos y demográficos de la población de estudio, y evaluar la relación de las diferentes variables con la recurrencia y la supervivencia a largo plazo de los pacientes.

\section{Materiales y métodos}

Se llevó a cabo un estudio descriptivo retrospectivo de una serie consecutiva de 97 hepatectomías por metástasis de cáncer colorrectal, en el período comprendido entre el 15 de junio de 2005 y el 30 diciembre de 2015 en el Hospital Pablo Tobón Uribe de Medellín.

La técnica quirúrgica de la transección hepática es diversa y se ha modificado a lo largo del tiempo; en sus primeros cinco años se utilizó la pinza de Kelly, luego, el bisturí ultrasónico y, posteriormente, el aspirador ultrasónico. Sólo se utiliza la maniobra de Pringle cuando es necesaria, con un esquema de 15 minutos de isquemia alternados con 5 minutos de reperfusión.

En los pacientes con metástasis sincrónicas, se decide el tipo de abordaje, clásico, sincrónico o el hígado primero, de acuerdo con el análisis individual de cada caso. El abordaje sincrónico no se utiliza para hepatectomías mayores con resecciones de recto, ni para resecciones mayores de colon. Cuando se calcula una función hepática remanente insuficiente, se opta por la generación de embolía preoperatoria de la vena porta.

En el Hospital, se utiliza el protocolo quirúrgico de recuperación rápida (Fast Track Surgery) con estancia hospitalaria de tres días (tabla 1), y los criterios de salida se basan en el protocolo ERAS (Early Recovery After Surgery) de resección hepática (tabla 2$)^{4}$.

Las variables cualitativas se describen en frecuencias absolutas y relativas en el análisis univariado. Las variables cuantitativas se expresan en medidas de tendencia central con las correspondientes medidas de dispersión.

TABLA 1.

Actividades que se deben realizar en los pacientes candidatos a 'salida rápida' (fast track), después de la hepatectomía

\begin{tabular}{l}
\hline Día anterior a la cirugía \\
\hline Nutrición normal \\
Sin líquidos intravenosos \\
\hline Día de la cirugía \\
\hline Restricción de líquidos \\
intravenosos \\
Presión venosa central menor \\
de 5 mmHg \\
Sin sonda gástrica de rutina \\
Líquidos intravenosos \\
calientes, manta térmica \\
Bloqueo del plano transverso \\
del abdomen \\
Líquidos orales 6 horas des- \\
pués de la intervención \\
Exámenes de laboratorio a \\
las 6 horas
\end{tabular}

\begin{tabular}{l}
\hline \multicolumn{1}{c}{ Día posquirúrgico 1 } \\
\hline Movilización \\
Retirar líquidos intravenosos \\
Dieta blanda \\
Exámenes de laboratorio \\
Retirar la sonda vesical \\
\hline Día posquirúrgico 2 \\
\hline Retirar el catéter central \\
Dieta blanda o normal \\
Mayor movilización \\
Exámenes de laboratorio \\
\hline Día posquirúrgico 3 \\
\hline Retirar el drenaje si lo tiene \\
Verificar los criterios de salida \\
\hline
\end{tabular}


TABLA 2.

Criterios que se deben tener en cuenta para dar alta a los pacientes después de la hepatectomía

Criterios de salida

Bilirrubina normal

Buen control del dolor

Tolerancia a la dieta

Movilización adecuada

No tener líquidos intravenosos

Se hizo el análisis bivariado de los factores asociados a la supervivencia. Para el análisis bivariado, se usaron las pruebas de ji al cuadrado para las variables cualitativas y, para las variables cuantitativas, la prueba t de Student si la distribución era normal, o la prueba U de Mann-Whitney si la variable no tenía distribución normal; la normalidad de las variables se determinaron por la prueba de Kolmogorov-Smirnov.

El análisis de supervivencia fue evaluado con el método de Kaplan-Meier y, las variables asociadas, con la prueba de log-rank.

\section{Resultados}

Entre el 15 de junio de 2005 y el 30 de diciembre de 2015, se practicaron 370 hepatectomías en el Servicio de Cirugía Hepatobiliar y Pancreática del Hospital Pablo Tobón Uribe, de las cuales 97 se debieron a metástasis de carcinoma colorrectal. Esta fue la indicación más frecuente de hepatectomía; le siguieron, en orden de frecuencia, el hepatocarcinoma, el cáncer de vesícula, la hepatolitiasis, las metástasis de tumores no colorrectales y no neuroendocrinos, el colangiocarcinoma y las metástasis de tumores neuroendocrinos.

Características de los pacientes. La edad promedio fue de 58 años y el 48,2 \% eran hombres. El régimen de seguridad social al que pertenecían los pacientes era el contributivo, el 66,7\%, el subsidiado, el 10,8 \% y la medicina prepagada, el 22,6\%.

Características del tumor. E1 $80 \%$ de los pacientes recibió quimioterapia preoperatoria. El 90,3\% de las metástasis fueron de cáncer de colon y, el 9,7\%, de cáncer de recto. Las metástasis fueron metacrónicas en $63,4 \%$ de los casos y sincrónicas en el 36,6\%. La mediana del número de metástasis fue de 2 (rango intercuartílico, RIQ: 1 a 3), con una mediana de tamaño de 3,5 cm (RIQ: 2,3 a $5 \mathrm{~cm}$ ). El $53 \%$ de las metástasis correspondía a una neoplasia maligna bien diferenciada, el 39,4 \%, a una moderadamente diferenciada, y el 7,6\% (con diferenciación buena o moderada) eran productoras de mucina. Se consiguió una resección 0 $\left(\mathrm{R}_{0}\right)$ en $84,4 \%$ de las hepatectomías y, una resección $1\left(R_{1}\right)$, en el $15,6 \%$.

Características quirúrgicas. Se practicó hepatectomía mayor (tres o más segmentos de Couinaud) en 50,5\% de los pacientes. El sangrado intraoperatorio tuvo una mediana de $200 \mathrm{ml}$ (RIQ: 100 a $400 \mathrm{ml}$ ). El 90,6\% de los pacientes no necesitó transfusión perioperatoria de hemoderivados, el 7,2\% recibió dos unidades de glóbulos rojos y solo en dos pacientes se usaron más de tres unidades de glóbulos rojos empaquetados. El tiempo quirúrgico tuvo una mediana de 150 minutos (RIQ: 120 a 180 minutos). Solo se trasladaron a la unidad de cuidados intensivos el 25,9\% de los pacientes y, de estos, el 67,9 $\%$ estuvo solo un día. Se usó el protocolo quirúrgico de recuperación rápida en $54,8 \%$ de los pacientes, los cuales tuvieron una estancia de tres o menos días. La estancia hospitalaria global tuvo una mediana de tres días (RIQ: 2 a 4 días).

Complicaciones. La morbilidad perioperatoria a 90 días fue de $21,5 \%$, algunos pacientes presentaron dos o más complicaciones, discriminadas así: infección del sitio operatorio, 7,5\%; fístula biliar, 2,2 \%; sangrado posoperatorio, 3,2 \%; infección a distancia, 4,3\%, y otras, $10 \%$; estas últimas incluyeron: derrame pleural, falla renal, hemotórax coagulado por catéter venoso central, obstrucción del trayecto gástrico de salida, íleo, y atelectasia y tromboembolia pulmonar. Según la clasificación de Clavien, 20,6 \% de las complicaciones fueron de grado I, $2,06 \%$, de grado II, y $2,06 \%$, de grado V. La mortalidad perioperatoria a 90 días fue de 2,06\% (un paciente por falla hepática posoperatoria y otro por sepsis secundaria a trombosis de la vena porta).

Supervivencia. El seguimiento ha sido hasta de 100 meses en esta serie, con una mediana de 20 meses. La supervivencia global a cinco años es de $34,5 \%$ y, a 100 meses, de $23 \%$ (figura 1 ). 
Análisis bivariado de supervivencia. La mediana de supervivencia para las metástasis sincrónicas fue de 45,5 meses (RIQ: 42,2 a 48,9 meses) y, la de las metástasis metacrónicas, de 57,9 meses (RIQ: 28,9 a 87 meses) $(p=0,962)$. La mediana de supervivencia para los pacientes con medicina prepagada fue de 48,6 meses (RIQ: 29,1 a 68,2 meses), comparada con la de los regímenes contributivo y subsidiado, de 45,5 meses (RIQ: 28,9 a $62,2$ meses) $(\mathrm{p}=0,965)$, (figura 2$)$.

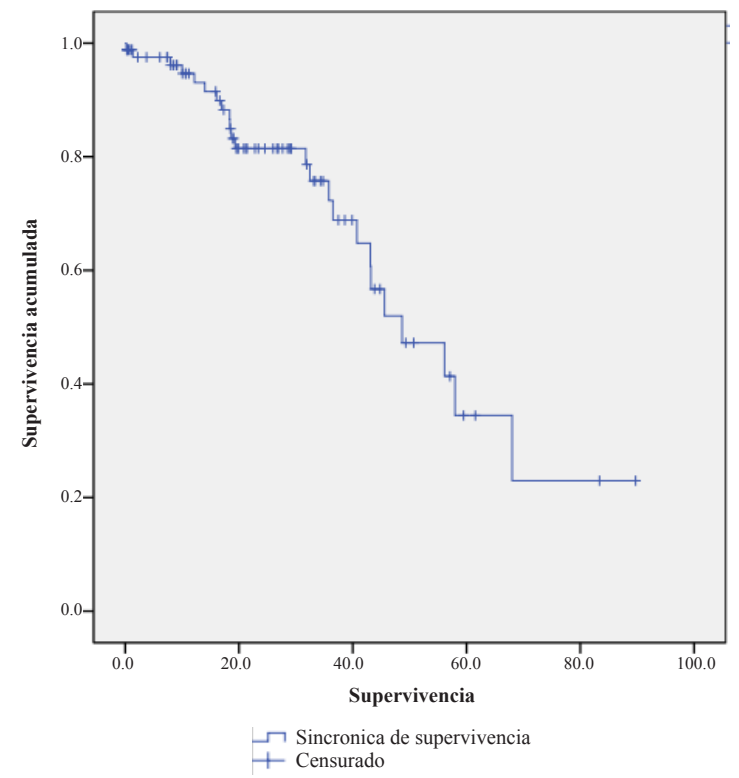

FIGURA 1. Supervivencia a largo plazo

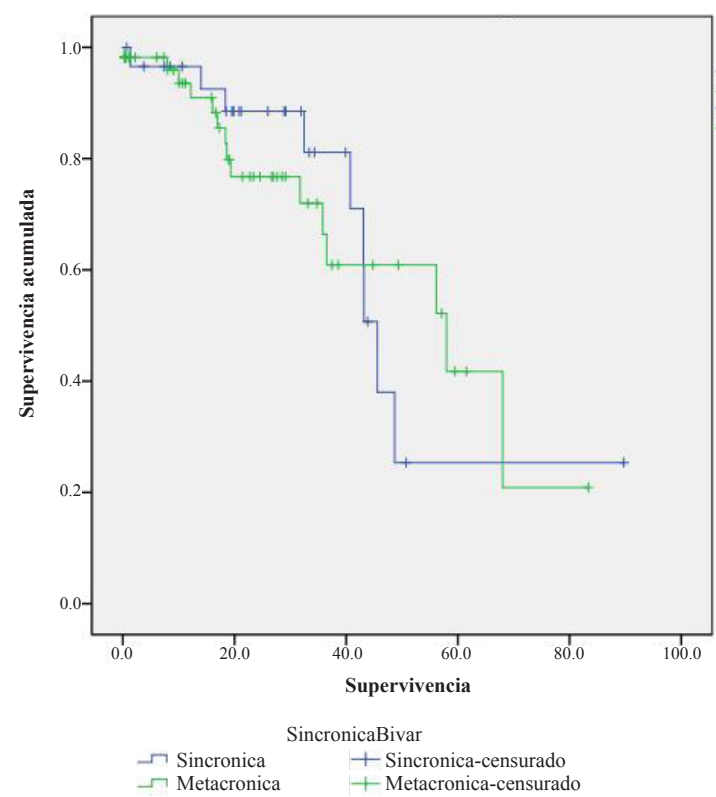

Figura 2. Supervivencia en metástasis sincrónicas y metacrónicas
El promedio de supervivencia para quienes recibieron quimioterapia previa fue de 42,7 meses (RIQ: 35,6 a 49,9 meses), comparada con la de los que no la recibieron, de 72,2 meses (RIQ: 53,5 a 90,9 meses) $(\mathrm{p}=0,012)$ (figura 3).

No hubo asociación entre un sangrado mayor de 500 $\mathrm{ml}$ y una menor supervivencia $(\mathrm{p}=0,653)$.

El promedio de supervivencia de quienes presentaron cualquier tipo de complicación fue de 52,8 meses (RIQ: 44 a 61 meses), comparado con el de quienes no presentaron ninguna, de 48,9 meses (RIQ: 39,4 a 58,3 meses) $(p=0,162)$.

El promedio de supervivencia para los menores de 50 años fue de 41 meses (RIQ: 33 a 49 meses), comparado con el de los mayores de 50 años que fue de 54 meses (RIQ: 43 a 65 meses) $(\mathrm{p}=0,466)$.

El promedio de supervivencia para quienes tenían el tumor primario en el recto fue de 41 meses (RIQ: 20,7 a 61,4 meses), comparado con el de quienes tenían el tumor primario en el colon que fue de 51,6 meses (RIQ: 42 a 61 meses $)(p=0,934)$ (tabla 3$)$.

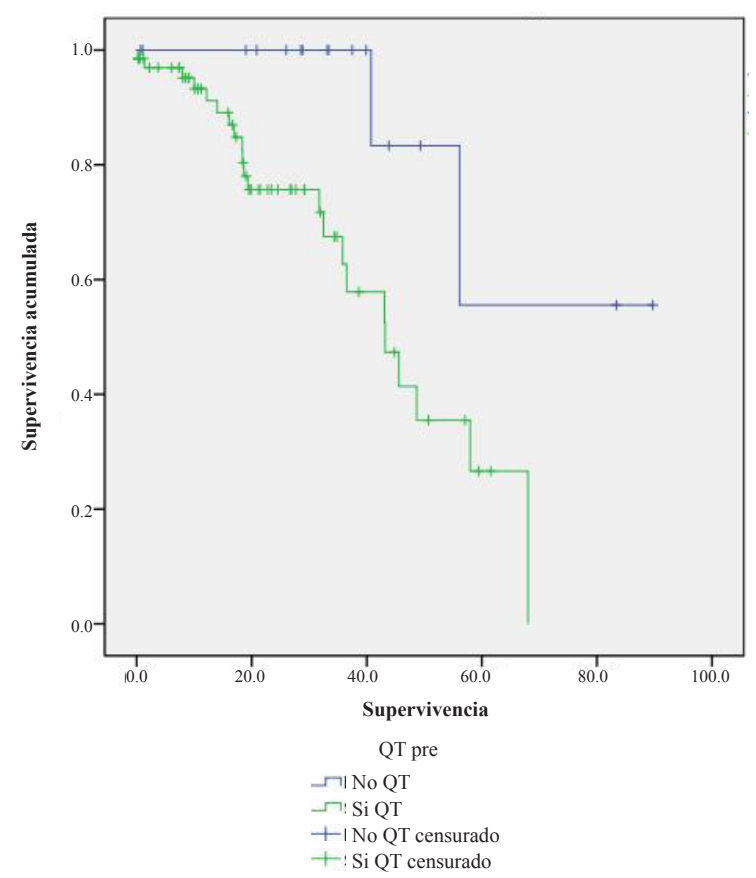

FIGURA 3. Supervivencia en pacientes que recibieron quimioterapia (QT) preoperatoria 
TABLA 3.

Análisis bivariado de variables demográficas, quirúrgicas y de anatomía patológica, en la supervivencia de los pacientes sometidos a resección de metástasis de carcinoma colorrectal, Hospital Pablo Tobón Uribe, 15 de junio de 2005 al 30 de diciembre 2015

\begin{tabular}{llll}
\hline \multicolumn{1}{c}{ Variable } & $\begin{array}{c}\text { Tiempo } \\
\text { (meses) }\end{array}$ & $\begin{array}{c}\text { Rango } \\
\text { intercuartílico } \\
\text { (meses) }\end{array}$ & p \\
\hline Metástasis sincrónica & 45,5 & $29,1-68,2$ & 0,162 \\
Metástasis metacrónica & 57,9 & $28,9-87$ & \\
\hline Póliza & 48,6 & $29,1-68,2$ & 0,965 \\
Contributivo/subsidiado & 45,5 & $28,9-62,2$ & \\
\hline Sin quimioterapia previa & 72,2 & $53,5-90,9$ & 0,012 \\
Con quimioterapia previa & 42,7 & $35,6-49,9$ & \\
\hline Complicación (cualquiera) & 52,8 & $44-61$ & 0,162 \\
Sin complicaciones & 48,9 & $39,4-58,3$ & \\
\hline Edad mayor de 50 años & 41 & $33-49$ & 0,466 \\
50 años o menos & 54 & $43-65$ & \\
\hline Recto & 41 & $20,7-61,4$ & 0,934 \\
Colon & 51,6 & $42-61$ & \\
\hline
\end{tabular}

El grado de diferenciación tumoral de la metástasis no tuvo injerencia en la supervivencia a largo plazo $(p=0,883)$, y tampoco el hecho de que la metástasis fuera única o múltiple ( $\mathrm{p}=0,312)$; incluso, la presencia de márgenes negativos en esta serie no afectó la supervivencia $(\mathrm{p}=0,946)$ (figura 4$)$.

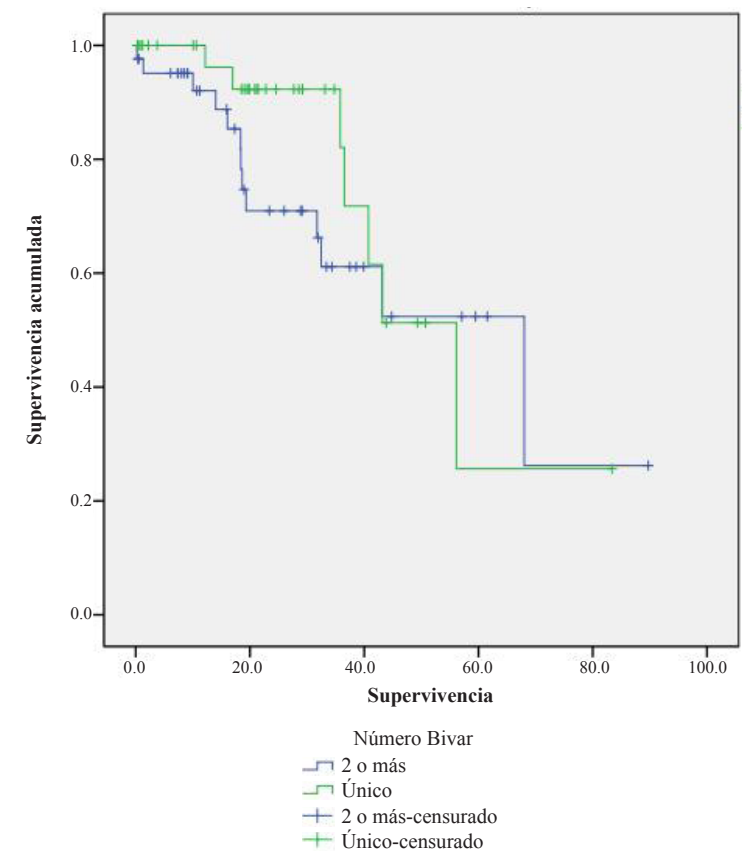

Figura 4. Supervivencia según número de metástasis
Debido a que no se encontró significancia estadística con las variables en el análisis bivariado para supervivencia, no se hizo análisis multivariado.

\section{Discusión}

La resección hepática fue establecida hace más de 20 años como el tratamiento de elección para las metástasis hepáticas resecables de cáncer colorrectal, y hay estudios de hace 40 años con supervivencia a cinco años cercana al $20 \%{ }^{5,6}$.

Con el refinamiento de la técnica quirúrgica de la resección hepática, los nuevos agentes quimioterapéuticos de las últimas dos décadas y los tratamientos no quirúrgicos dirigidos al hígado, como la ablación por radiofrecuencia, se han ampliado las indicaciones para hepatectomía en metástasis de cáncer colorrectal, lográndose tratar pacientes con metástasis que antes no podían resecarse y alcanzándose supervivencias globales a cinco años de 25 a $44 \%{ }^{7,8}$.

En esta serie se practicó hepatectomía mayor en la mitad de los pacientes, el sangrado intraoperatorio fue bajo (mediana de $200 \mathrm{ml}$ ) y en la gran mayoría no se necesitó transfusión perioperatoria de hemoderivados. Solo se trasladaron a la unidad de cuidados intensivos en el posoperatorio el $25 \%$ de los pacientes y, de estos, la mayoría estuvo solo un día. En 54,8 \% de los pacientes se cumplió con el protocolo quirúrgico de recuperación rápida, en el cual se contempla una estancia hospitalaria de tres o menos días; este es uno de los protocolos con estancia más corta reportados en la literatura científica ${ }^{9}$.

Según el tipo de aseguramiento en salud, se han encontrado diferencias en el resultado del tratamiento, el cual es mejor en el tipo privado que en el público; estos resultados se han obtenido en estudios de enfermedades oncológicas y no oncológicas, quirúrgicas y no quirúrgicas, en países desarrollados y pobres, y en cirugía hepática ${ }^{10}$. No hubo diferencia en la supervivencia de los pacientes con medicina prepagada, al comparar esta con los regímenes contributivo y subsidiado, a los que pertenece la mayoría de los pacientes atendidos en el hospital.

La mayoría de las metástasis resecadas fueron de cáncer de colon; la supervivencia para estos pacientes fue mejor que para aquellos con metástasis de cáncer 
de recto, aunque esto no alcanzó una significancia estadística.

En concordancia con la literatura científica mundial, la mediana de supervivencia en casos de metástasis metacrónicas fue mayor que en los de metástasis sincrónicas (57,9 Vs. 45,5 meses); sin embargo, la diferencia no fue estadísticamente significativa ${ }^{7,8,11}$.

En esta serie, la mayoría de los pacientes recibió quimioterapia preoperatoria. Llama la atención que su supervivencia fue mayor que la de quienes no la recibieron; la diferencia fue estadísticamente significativa $(\mathrm{p}=0,012)$. Esto podría relacionarse con un estado más avanzado de la enfermedad, pues el paciente recibe quimioterapia adyuvante cuando las metástasis se diagnostican y ya no pueden resecarse; además, hay demoras para la aprobación y la administración de los esquemas de quimioterapia, y también, para la remisión a cirugía radical, por deficiencias del sistema de salud.

El funcionamiento del sistema de seguridad social en salud de Colombia no permite controlar variables como la elección de la quimioterapia neoadyuvante o adyuvante. Las aseguradoras fragmentan la atención de los pacientes y los remiten para la quimioterapia a otros hospitales de acuerdo con su red de contratación, por lo cual es imposible para los médicos tratantes protocolizar esquemas de quimioterapia. En muchos casos, esta fragmentación arruina los planes de tratamiento de hepatectomía en etapas, quimioterapia neoadyuvante y terapia multimodal dirigida al hígado. Otros factores de riesgo en casos de metástasis, que tradicionalmente se consideran para la supervivencia a largo plazo, como diferenciación celular, tamaño y número de las metástasis, no alcanzaron una significancia estadística en el presente estudio ${ }^{12,13}$.

La morbilidad perioperatoria a 90 días fue de $21,5 \%$, la mayor parte $(20,6 \%)$ de las complicaciones quirúrgicas fueron de grado I según la clasificación de Clavien-Dindo y la mortalidad perioperatoria a 90 días fue de 2,06 \%, datos similares a los de otros reportes a nivel mundial ${ }^{14,15}$.

El resultado oncológico a largo plazo también estuvo acorde con el de otros estudios, con una supervivencia global de $34,5 \%$ a cinco años y de $23 \%$ a 8,3 años ${ }^{2,3,12}$.

Las limitaciones de este estudio son su carácter retrospectivo y la dificultad constante en nuestro medio para hacer un seguimiento posquirúrgico adecuado a causa de la constante migración de los pacientes entre diferentes centros; esta se debe a la contratación de las aseguradoras, cuya única prioridad es reducir costos, sin importarles otros aspectos fundamentales en la adecuada atención de enfermedades complejas, como las neoplasias malignas.

En conclusión, la resección hepática en pacientes con metástasis de cáncer colorrectal en nuestro medio se practica de una manera segura, con morbilidad, mortalidad y resultado oncológico a largo plazo comparables con los de otras series del mundo.

\section{Agradecimientos}

Este estudio hace parte del proyecto financiado por el Departamento Administrativo de Ciencia, Tecnología e Innovación, Colciencias (Código: 111556935008).

\title{
Hepatic resection of metastases in colorectal cancer, survival analysis in a cohort of patients at Pablo Tobón Uribe Hospital, Medellín, Colombia
}

\begin{abstract}
Introduction. Hepatic resection remains the only treatment option with curative intent and long-term survival in colorectal liver metastasis. The aim of this study is to evaluate the outcomes of hepatic resection in this group of patients.

Methods. A retrospective descriptive study of a consecutive series of 97 hepatectomies due to colorectal cancer metastases in the period between June 2005 and December 2015 at the Pablo Tobón Uribe Hospital in Medellin. Bivariate analysis was performed on factors associated with survival. Results. Of a total of 370 hepatectomies, 97
\end{abstract}


liver resections were performed in 93 patients due to colorectal cancer metastases. The mean age was 58 years, $50.5 \%$ were major liver resections. The median intraoperative bleeding was $200 \mathrm{ml}$ (RIQ 100-400 $\mathrm{ml}$ ) and the median surgical time was 150 minutes (RIQ 120-180 min). The hospital stay had a median of 3 days (RIQ 2-4 days). Only 25.9\% of the patients were transferred to the intensive care unit. Complications occurred in $21.5 \%$ of patients. Perioperative mortality at 90 days was $2.06 \%$. A fast track protocol was performed in $54.8 \%$ of the patients. The 5-year actuarial survival was $34.5 \%$ and at 100 months it was 23\%. Because no statistical significance was found with any of the variables in the bivariate analysis for survival, no multivariate analysis was performed.

Conclusions. Liver resection in patients with colorectal cancer metastases can safely be performed in our setting, with morbidity, mortality and cancer outcomes comparable to the other series in the world.

Key words: Neoplasms; liver; colonic neoplasms; neoplasm metastasis; hepatectomy; survival.

\section{Referencias}

1. Adam R, De Gramont A, Figueras J, Guthrie A, Kokudo N, Kunstlinger $\mathrm{F}$, et al. The oncosurgery approach to managing liver metastases from colorectal cancer: A multidisciplinary international consensus. Oncologist. 2012;17:1225-39.

2. Mayo SC, Pulitano C, Marques H, Lamelas J, Wolfgang CL, de Saussure W, et al. Surgical management of patients with synchronous colorectal liver metastasis: A multicenter international analysis. J Am Coll Surg. 2013;216:707-16.

3. Adam R, Pascal G, Castaing D, Azoulay D, Delvart V, Paule $\mathrm{B}$, et al. Tumor progression while on chemotherapy: A contraindication to liver resection for multiple colorectal metastases? Ann Surg. 2004;240:1052-61.

4. van Dam RM, Hendry PO, Coolsen MM, Bemelmans MH, Lassen K. Initial experience with a multimodal enhanced recovery programme in patients undergoing liver resection. $\mathrm{Br} \mathrm{J}$ Surg. 2008;95:969-75.

5. Wilson SM, Adson MA. Surgical treatment of hepatic metastases from colorectal cancers. Arch Surg. 1976;111:330-4.

6. Manfredi S, Lepage C, Hatem C, Coatmeur O, Faivre J, Bouvier AM. Epidemiology and management of liver metastases from colorectal cancer. Ann Surg. 2006;244:254-9.

7. Neumann UP, Thelen A, Röcken C, Seehofer D, Bahra M, Riess $\mathrm{H}$, et al. Non-response to pre-operative chemotherapy does not preclude long-term survival after liver resection in patients with colorectal liver metastases. Surgery. 2009;146:52-9.

8. Cummings LC, Payes JD, Cooper GS. Survival after hepatic resection in metastatic colorectal cancer: A population-based study. Cancer 2007;109:718-26.

9. Song W, Wang K, Zhang RJ, Dai QX, Zou SB. The Enhanced Recovery After Surgery (ERAS) program in liver surgery: A meta-analysis of randomized controlled trials. Springerplus. 2016;29:207-13.
10. Zaydfudim V, Whiteside MA, Griffin MR, Feurer ID, Wright JK. Health insurance status affects staging and influences treatment strategies in patients with hepatocellular carcinoma. Ann Surg Oncol. 2010;17:3104-11.

11. Adam R, de Gramont A, Figueras J, Kokudo N, Kunstlinger F, Loyer E, et al. Managing synchronous liver metastases from colorectal cancer: A multidisciplinary international consensus. Cancer Treat Rev. 2015;41:729-41.

12. Mekenkamp LJ, Koopman M, Teerenstra S, van Krieken JH, Mol L, Nagtegaal ID, et al. Clinicopathological features and outcome in advanced colorectal cancer patients with synchronous vs. metachronous metastases. Br J Cancer. 2010;103: 159-64.

13. Conrad C, You N, Vauthey JN. In patients with colorectal liver metastases, can we still rely on number to define treatment and outcome? Oncology (Williston Park). 2013;27:1078

14. Dindo D1, Demartines N, Clavien PA. Classification of surgical complications: A new proposal with evaluation in a cohort of 6336 patients and results of a survey. Ann Surg. 2004;240: 205-13.

15. De Santibañes E, Lassalle FB, McCormack L, Pekolj J, Quintana GO, Vaccaro C, et al. Simultaneous colorectal and hepatic resections for colorectal cancer: postoperative and longterm outcomes. J Am Coll Surg. 2002;195:196-202.

Correspondencia: Sergio Hoyos, MD

sergiohoyosd@yahoo.es

Medellín, Colombia 